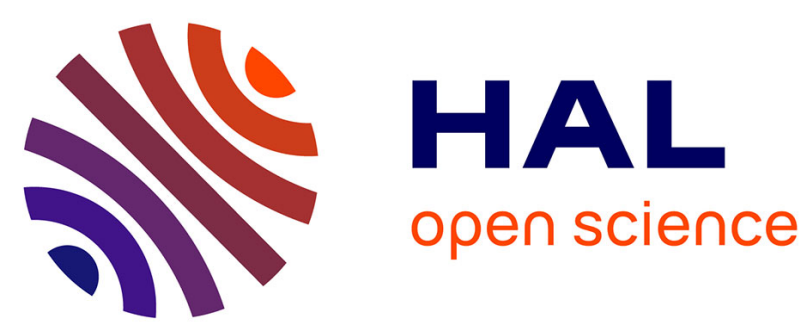

\title{
Interactive Simulation of Diaphragm Motion Through Muscle and Rib Kinematics
}

\author{
Pierre-Frédéric Villard, Wesley Bourne, Fernando Bello
}

\section{To cite this version:}

Pierre-Frédéric Villard, Wesley Bourne, Fernando Bello. Interactive Simulation of Diaphragm Motion Through Muscle and Rib Kinematics. Nadia Magnenat-Thalmann, Jian J. Zhang, David D. Feng. Recent Advances in the 3D Physiological Human, Springer London, pp.91-103, 2009, 10.1007/978-184882-565-9_6. hal-00430234

\section{HAL Id: hal-00430234 https://hal.science/hal-00430234}

Submitted on 6 Nov 2009

HAL is a multi-disciplinary open access archive for the deposit and dissemination of scientific research documents, whether they are published or not. The documents may come from teaching and research institutions in France or abroad, or from public or private research centers.
L'archive ouverte pluridisciplinaire HAL, est destinée au dépôt et à la diffusion de documents scientifiques de niveau recherche, publiés ou non, émanant des établissements d'enseignement et de recherche français ou étrangers, des laboratoires publics ou privés. 


\title{
Interactive Simulation of Diaphragm Motion Through Muscle and Rib Kinematics
}

\author{
Pierre-Frédéric Villard ${ }^{1}$, Wesley Bourne ${ }^{2}$ and Fernando Bello ${ }^{3}$
}

\begin{abstract}
Modelling of diaphragm behaviour is of relevance to a number of clinical procedures such as lung cancer radiotherapy and liver access interventions. The heterogeneity in tissue composition of the diaphragm, as well as the various physiological phenomena influencing its behaviour, requires a complex model in order to accurately capture its motion. In this paper we present a novel methodology based on a heterogeneous model composed of mass-spring and tensegrity elements. The physiological boundary conditions have been carefully taken into account and applied to our model. Thus, it incorporates the influence of the rib kinematics, the muscle natural contraction/relaxation and the motion of the sternum. Initial validation results show that the behaviour of the model closely follows that of a real diaphragm.
\end{abstract}

\section{Introduction}

Diaphragm motion has a crucial influence on surrounding organs. Real time knowledge of its behaviour is of great significance in the context of various treatments for procedure rehearsal, planning and guidance. We are particularly interested in lung cancer radiotherapy and liver access procedures. In the first case, the lung is subject to the up and down movement of the diaphragm, which can in turn produce tumour movement of up to $5 \mathrm{~cm}$ [1]. Liver motion is an important factor to consider in liver access procedures. It has been estimated to be up to $3 \mathrm{~cm}$ [2] Such motion is directly related to diaphragm movement, which is transmitted to the liver through its ligamentous attachments.

\footnotetext{
${ }^{1}$ Biosurgery and Surgical Technology, Imperial College London, UK p.villard@imperial.ac.uk

${ }^{2}$ Biosurgery and Surgical Technology, Imperial College London, UK wesley.bourne@morganstanley.com

${ }^{3}$ Biosurgery and Surgical Technology, Imperial College London, UK f.bello@imperial.ac.uk
} 
A possible solution to predict diaphragm motion is to apply displacement vectors obtained from non-rigid registration in combination with a statistical approach as in von Siebenthal et al [3]. A disadvantage of this approach is that it tends to assume a reproducible breathing cycle, which has been demonstrated to be not always a valid assumption [1]. Our aim is to build a model that can be customized to the patient to comply with the accuracy requirements of radiotherapy. Physicallybased models of the diaphragm have already been proposed in the past (e.g. $[4,5]$, etc.), but they are mainly based on mass-spring systems, with their motion dictated by a single parameter.

We proposed in [6] a model that takes into account the heterogeneity of the diaphragm, considering its main tissues types, as well the relevant physiological phenomena and various forces involved during normal diaphragm movement, thus introducing additional parameters to fine tune / control the motion. It consists of mass-spring elements for the muscular part and Tensegrity elements [7] for the tendinous part. Here we focus on the respiration process. Deformations are produced by three different phenomena: the diaphragm contraction, the ribs kinematics and the sternum motion. This paper presents our methodology and initial validation results on a commercial virtual anatomy model, two radiotherapy patients and a normal volunteer.

\section{Materials and methods}

We start with a careful study of the relevant anatomy and physiology as it is important to understand the real behaviour of the diaphragm in order to be able to establish adequate boundary conditions and select suitable physical models. This section also indicates the integration technique used and the method to monitor the external forces.

\section{Anatomy and physiology}

Research on previous work about diaphragm physiology was focused on its anatomical, histological and mechanical components. The data obtained were analyzed and compared to determine viability and validity for its use. Based primarily on the work presented in $[8,9,10]$, we obtained the main physiological components involved in displacement of the diaphragm and studied how each one of them influences diaphragm motion. 

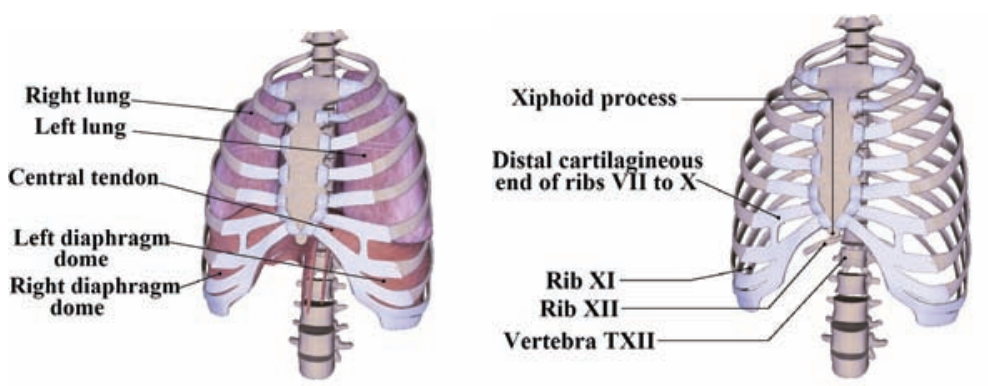

Fig. 1. Left: Thorax anatomy and right: Attachment points of the diaphragm.

The thoracic diaphragm is a domed fibromuscular sheet that separates the thoracic and abdominal cavities. It is composed of two domes and the right dome is higher than the left dome (Fig. 1 left). The peripheral part of the diaphragm consists of muscular fibres that converge on a central tendon at the level of the xiphisternum. There are three different groups of muscular fibres:1) Sternal, attached to the back of the xiphoid process 2) Costal, attached on the inner surfaces of cartilages and adjacent portions of lower six ribs on either side and 3) Lumbar, attached to the lumbar vertebrae (Fig. 1 right).

Given the spatial dimension, topology and tissue composition of the diaphragm, it can be modelled as a heterogeneous surface composed of muscle and tendon. The origins of the muscle fibres define the attachment points of the diaphragm. They are used to define the boundary conditions for our model as follows: 1) The xiphoid process of the sternum, 2) The costal margin of thoracic wall (lower 6 ribs and their costal cartilages), 3) The end of ribs XI and XII, and 4) The vertebrae of lumbar region. We call them the attachment points and the details of the implementation will be described in the following section.

\section{Deformation Modelling}

In their survey of deformable models for surgery simulation, Meier et al. [11] claim that mass-spring systems and Boundary Element Models (BEM) are the best models for surgical simulators because they allow a realistic behaviour while supporting near real time performance. Thalmann and Nedel [12] proposed a model of muscle based on a surface mass-spring system oriented according to the muscular fibre calling it the "action line". We borrow from this formulation the use of a predefined unique line to parameterise the action of a muscle, as well as the massspring formulation, in order to model the muscular component of the diaphragm. Concerning the tendinous part, which can be described as a tough band of fibrous connective tissue, we use a tensegrity model as proposed by Inger to model certain parts of the body [7]. 
The word "tensegrity" comes from a contraction of "Tensional integrity". It is used to define a mechanical system with components that combine tension and compression in such a way as to enable the whole system to receive and apply forces, tensions and pressures. Tensegrity systems are composed of two kinds of elements: the elastic elements, which give the system tension, and the rigid elements, which have a constant length and that will exert compression forces.

The evolution of node positions is simulated by taking into account all of its links that could either be elastic or rigid. Each point is represented at a time $t$ by the triplet $(a(t), v(t), p(t))$ representing the acceleration, velocity and position. As we define the forces to apply on the nodes with elastic and rigid links, we can calculate their acceleration with the fundamental law of dynamics:

$$
\sum \mathbf{f}=m \cdot \mathbf{a}
$$

It is then possible to compute the velocity and the position at time $(t+\Delta t)$ :

$$
\left\{\begin{array}{c}
\mathbf{v}(\mathrm{t}+\Delta \mathrm{t})=\int_{t}^{\mathrm{t}+\Delta \mathrm{t}} \sum_{t}^{\mathrm{t}+\Delta \mathrm{t}} \mathbf{f} / m d t+\mathbf{v}(t) \\
\mathrm{p}(\mathrm{t}+\Delta \mathrm{t})=\int_{t}^{\mathbf{v}} \mathbf{v}(t) d t+\mathrm{p}(t)
\end{array}\right.
$$

The elastic links are simulated as in the mass-spring system with two parameters: their initial length $\mathbf{l}_{\mathbf{0}}$ and their elasticity $k$. A force $\mathbf{F}_{\boldsymbol{e}}$ exerting on an elastic link to elongate its length from $\mathbf{I}_{\mathbf{0}}$ to $\mathbf{l}$ is thus expressed as follows:

$$
\mathbf{F}_{\mathrm{e}}=-\mathrm{k}\left(\mathrm{I}-\mathrm{I}_{0}\right)
$$

We add a damping force $F_{\mathbf{d}}$ to systematically and progressively decrease the velocity of each node.

$$
\mathbf{F}_{\mathrm{d}}=-\gamma \cdot \mathbf{v}
$$

The rigid links in the tensegrity part exert reaction forces to avoid changes in their length. We assume that there is no collision between rigid links. For each time step:

1. The elastic forces $\mathbf{F}_{\mathbf{e}}$ are computed using Eq. (3) as well as the new positions of the nodes as if there were no rigid links; 
2. The rigid constrains are applied to ensure that the distance between two nodes remains constant;

3. Given two nodes $\mathbf{A}$ and $\mathbf{B}$ linked by a rigid link, $\mathbf{A}^{\prime}$ and $\mathbf{B}$ ' are their respective positions after applying the classical mass-spring algorithm (as if there was no rigid link). The real positions $\mathbf{A}_{\mathbf{1}}$ and $\mathbf{B}_{\mathbf{1}}$ considering the rigid links are given by the following:

$$
\mathbf{A A 1} / \mathbf{B B}_{1}=\mathbf{B}_{1} \mathbf{B}^{\prime} / \mathbf{A}_{1} \mathbf{A}^{\prime}
$$

such that $\mathbf{A}_{\mathbf{1}}$ and $\mathbf{B}_{\mathbf{1}}$ remains on the line $\mathbf{A}^{\prime} \mathbf{B}^{\prime}$.

4. The new velocity is computed at each node.

The resulting set of differential equations is solved using a second order Runge-Kutta method:

$$
\left\{\begin{array}{c}
\mathbf{a}(t+\Delta t)=\sum \mathbf{f} \\
\mathbf{v}(t+\Delta t / 2)=1 / 2 \mathbf{a}(t-\Delta t) \Delta t+\mathbf{v}(t) \\
\mathbf{v}(t+\Delta t)=1 / 2 \mathbf{a}(t-\Delta t) \Delta t+\mathbf{v}(t+\Delta t / 2) \\
\mathbf{p}(t+\Delta t)=\mathbf{v}(t+\Delta t)+\mathbf{p}(t)+1 / 2 \mathbf{a}(t-\Delta t) \Delta t^{2}
\end{array}\right.
$$

We will now focus on the movement of the diaphragm during inhalation. First, the anterior-posterior diameter increases because the sternum moves forwards as ribs are raised. Secondly, the transverse diameter increases due to a "pump handle" movement which elevates the ribs. Both movements can be simulated by the combined motion of the ribs, the sternum and the cartilage. We first assume that the ribs are rigid and their motion can be modelled by a kinematics law based on the finite helical axis method as in [13]. The cartilage-sternum follows the motion of the ribs.

The attachment points are modelled by a mass-spring system with a very low elasticity in order to ensure that it remains attached. A fall-off function insures that the deformation field is continuous and smooth. The ones belonging to the diaphragm are automatically obtained such that the distance to the group \{ribs, sternum, cartilage is under a given threshold. Then, the vertical dimension increases due to diaphragm movement during inhalation. This motion can be simulated by the muscle action generation. We define an action line as a vertical line (inferiorsuperior) passing through given points and with a certain radius of action. The forces of contraction during inhalation or relaxation during exhalation are computed such that their intensity varies according to the distance from the action line. 


\section{Results}

We now present the purposely written simulation software. We have applied our methodology to a virtual anatomy model and three patients. The focus for the former was on the realism of the motion and especially on the influence of the parameters described previously, whereas for the later we compared our simulated displacements with the real motion measured on a 4D CT scan.

\section{Diaphragm Deformation Framework}

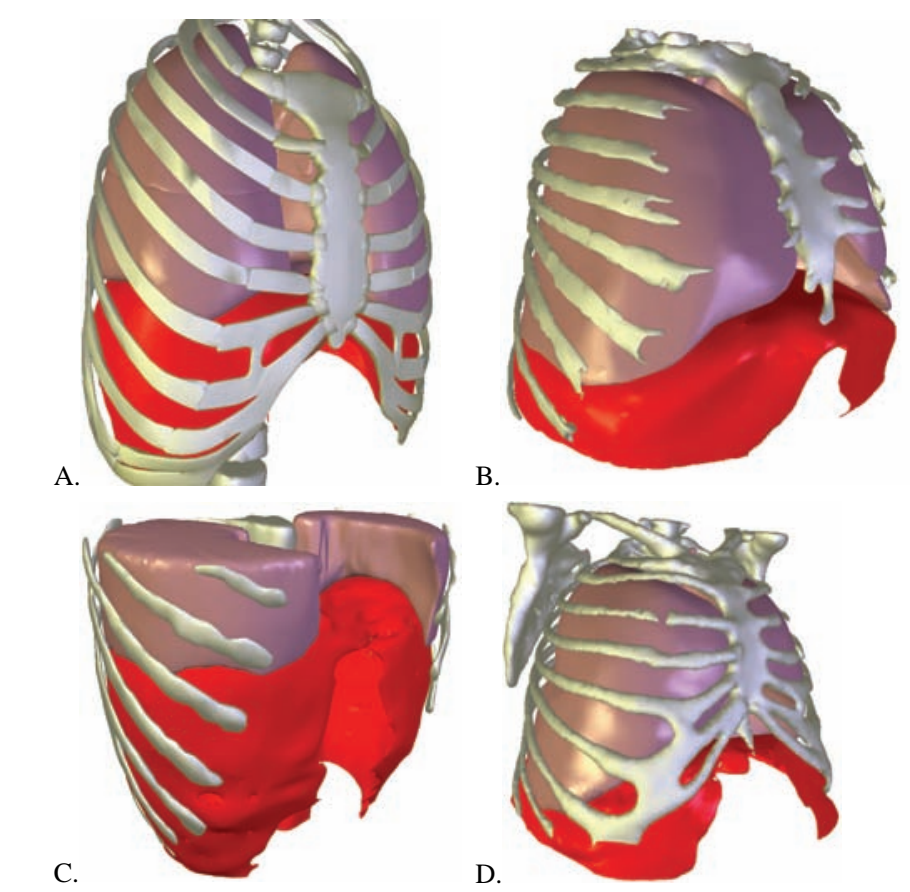

Fig. 2. Interface display of ribcage, lungs and diaphragm a) model b,c and d): patients.

Our framework is coded using Java 3D API ${ }^{4}$. It allows the real-time fine tuning of simulation parameters in order to study various types of breathing, breath hold, ribs rotation, diaphragm muscle force intensity, etc. The deformation framework enables the visualization of the following elements: 1) External skin by transpar-

${ }^{4}$ http://java.sun.com/products/java-media/3D/ 
ency (rigid model), 2) The spine (rigid model), 3) The ribs (modelled following a kinematics law), 4) The sternum and the ligaments attached to the ribs (modelled by a very stiff mass-spring system), and 5) The diaphragm (modelled as described above). Fig. 2 shows an illustration of the organ deformation display with examples of anatomy: a commercial virtual anatomy model and three real patients. The parameters of the simulation are: Damping $=-0.02$, Stiffness $=15$, mass point $=0.1$ and time step $=0.01$. Results of the simulation and validation using both models are now presented.

\section{Simplified model}

We used a simplified model from a detailed commercial anatomy set (Anatomium 3D by CF Lietzau 3D Special Service). The anatomical parts are shown in Fig. 1. The diaphragm was remeshed to obtain a surface mesh composed of 11359 nodes and 22722 triangles. The tendinous tissue and the muscle tissue were separated by a plane. The rigid links of the tensegrity part were created by linking the upper surface to the lower surface with a heuristic algorithm. The reasoning behind it is as follows. Once a suitable distance has been chosen, a list of close masses is found. These masses could either be neighbouring masses on the same surface or ideally those on opposing surfaces. Testing the angle removes those on the same surface, leaving a sorted list of masses on the opposing surface.

Fig. 3 is an illustration of the simulation results. The diaphragm geometry at the beginning of the simulation (end of inhale) is represented by a wireframe. The final geometry (end of exhale) is represented by the coloured surface. It can be noticed that the tendon has kept its smooth geometry due to the tensegrity elements, while the muscle part has extended more due to their elastic elements. The influence of the three phenomena mentioned before can be seen here: the muscle relaxation that increases the height of the domes, the ribs rotation that presses the diaphragm on each side and the sternum going backward that pushes the diaphragm.

Now that the realism of the simulations has been evaluated, we focus on the computational cost of the simulation parameters. The tests below were performed on a dual core $2.4 \mathrm{ghz}$ machine with 2 gigabytes of ram and a 256 megabyte graphics card. The operating system was Windows Vista Service Pack 1 with Java 1.6 installed. 


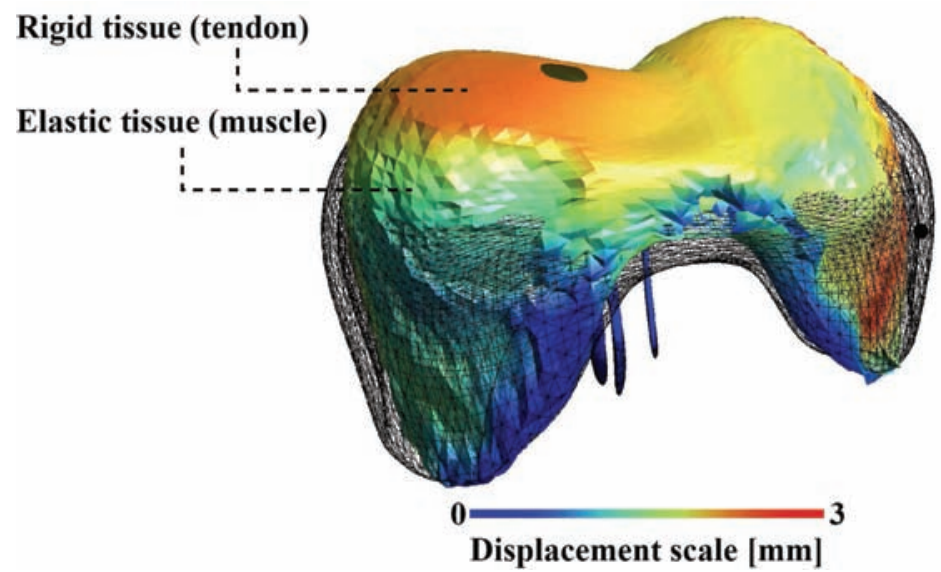

Fig. 3. Illustration of diaphragm motion during inhale.

Table 1 provides a succinct summary of all the data. It gives the average iterations per second after five trials. The relationship between the parameters of the simulation and the mesh size are available. The computational time of the rib kinematics is shown to be quite high as when they are removed in the "Diaph.muscle" trial the average iterations per second hits its peak. Regarding the cost on the speed of the simulator by the addition of tensegrity, it shows that the largest change is visible in the high quality perfect model. This is due to the very high number of connections created in this case.

Table 1. Iterations per second of all the simulations on the virtual anatomy model. The trials have been averaged into a single value.

\begin{tabular}{llllll}
\hline \multicolumn{2}{l}{ Resolution Vertices } & $\begin{array}{l}\text { Heterogenous (All- } \\
\text { Forces) }\end{array}$ & $\begin{array}{l}\text { Homogenous (All- } \\
\text { Forces) }\end{array}$ & $\begin{array}{l}\text { Ribsmotion } \\
\text { (Heterogenous) }\end{array}$ & $\begin{array}{l}\text { Diaph.muscle } \\
\text { (Heterogenous) }\end{array}$ \\
\hline High & 20909 & 8.13 & 8.56 & 8.24 & 9.68 \\
Medium & 16023 & 10.41 & 10.69 & 10.93 & 11.76 \\
Low & 12047 & 12.79 & 13.07 & 13.27 & 14.70 \\
\hline
\end{tabular}

Table 2. Iterations per second of all the simulations on a real patient model (subject 1). The trials have been averaged into a single value.

\begin{tabular}{llllll}
\hline Resolution Vertices & $\begin{array}{l}\text { Heterogenous (All- } \\
\text { Forces) }\end{array}$ & $\begin{array}{l}\text { Homogenous (All- } \\
\text { Forces) }\end{array}$ & $\begin{array}{l}\text { Ribsmotion } \\
\text { (Heterogenous) }\end{array}$ & $\begin{array}{l}\text { Diaph.muscle } \\
\text { (Heterogenous) }\end{array}$ \\
\hline High & 20909 & 8.13 & 8.56 & 8.24 & 9.68 \\
Medium & 16023 & 10.41 & 10.69 & 10.93 & 11.76 \\
Low & 12047 & 12.79 & 13.07 & 13.27 & 14.70 \\
\hline
\end{tabular}




\section{Patient specific models}

Our method can be used on real patients. To test it, we first took 2 sets of 4D CT scan data acquired during normal tidal breathing, therefore the diaphragm motions were not as large as in the virtual model described above. We then acquired 2 MRI datasets from a volunteer subject at two different levels of breath holding.

The diaphragms were manually segmented using ITK-SNAP 5 . A mesh was then generated using the CGAL library ${ }^{6}$ with a mesh angle of 5, mesh distance of 1 , and mesh radius of 1 . The mesh was smoothed using vtkWindowedSincPolyDataFilter() with a pass band of 0.2 , followed by a $501 \%$ decimation, followed by another 200 iterations of Windowed Sinc smoothing. An example of a segmented and meshed diaphragm is presented on Fig. 4. The mesh is composed of 6633 nodes and 13267 triangles.
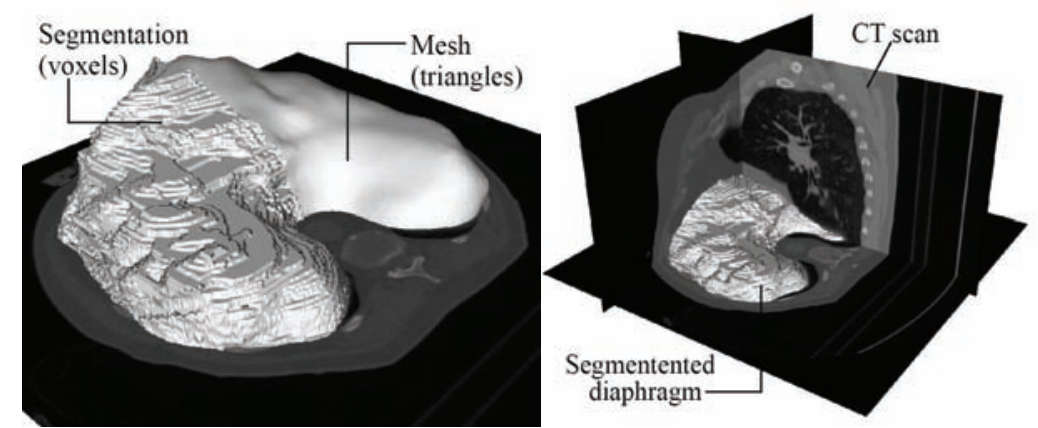

Fig.4. Left: Segmented diaphragm inside CT scan and right: Combination of segmentation and the mesh.

The rib cage and the lungs were segmented by the levelset method. Rib motion can be neglected in this case because of the normal tidal breathing and the position of the patient lying down on the scanning table. This assumption has been validated by computing the displacement of the ribs. Therefore, only the diaphragm relaxation needs to be taken into account from the initial exhale to the final exhale. The diaphragm will be attached (as in the virtual model) to the locations described in §2. The simulation is applied with small forces on the previously defined action line and the lungs are linked to the diaphragm with springs.

The simulation was compared to the imaging data at the same point in the breathing cycle by exporting the resulting lung geometry from the fine-tuned simulation and creating a mesh from the segmented 4D CT diaphragm. The differences were then measured and studied with the MESHDEV ${ }^{7}$ software that com-

\footnotetext{
${ }^{5}$ http://www.itksnap.org/

${ }^{6}$ http://www.cgal.org/

${ }^{7}$ http://www.meshdev.sourceforge.net/
} 
putes the distance between two triangular meshes. The result can be seen on Fig. 5. The image on the left represents the distance between the surface of the lungs from the CT scan at beginning and end of inhale. The image on the right represents the distance between the simulated end of inhale and the end of inhale extracted from medical images. Finally, Table 2 provides a succinct summary of the computing time. It can be seen that it is slower than the virtual simulator, attributed to the high count of vertices involved in the simulation.

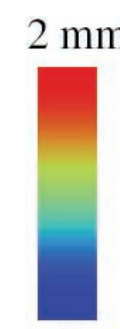

0

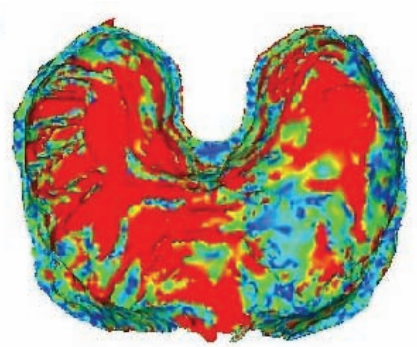

Subject 1

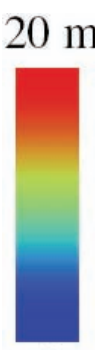

0
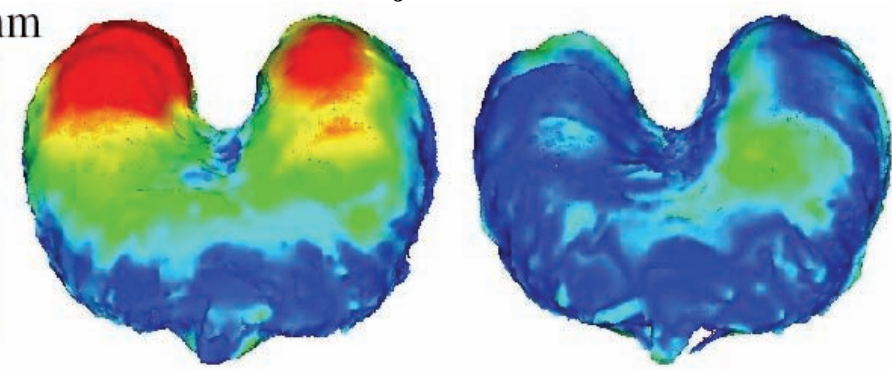

Subject 2

\section{$25 \mathrm{~mm}$}

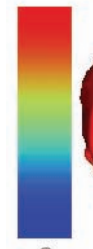

0
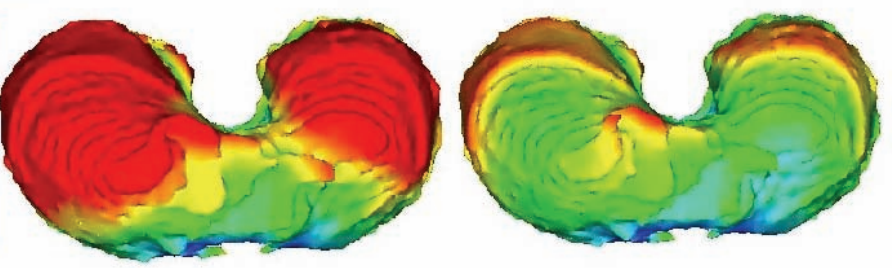

\section{Subject 3}

Fig. 5. Distance error measurement on the diaphragm between left: beginning and end of real inhale and right: simulated end of inhale and real end of inhale. 


\section{Discussion}

The physiological behaviour of this generic model was discussed with our clinical collaborator, who validated that the antero-posterior, transverse and vertical dimensions of the thorax are increased as illustrated by the model. It was also pointed out that only the domes descend when breathing lightly, whereas with a deep breath, the domes descend further and the central tendon can move from the level of vertebrae T8 to T9. In full exhale, the right dome reaches the 4th intercostal space and the left dome reaches the 5th rib. This complex behaviour further justifies the need for a heterogenous model such as that presented here.

Concerning the patient specific model, it can be seen that the main output of the simulation is the relaxation of the two domes. The contribution is only slightly significant on Subject 1 . This is mainly due to the diaphragm displacement (beginning to end of inhale) which is not large enough to correctly appreciate the advantage of the simulation. This displacement is much larger with the 4D CT scan of Subject 2 and with the MRI of Subject 3. The average diaphragm displacements are, respectively, $5.8 \mathrm{~mm}$ with a standard deviation of $5 \mathrm{~mm}$ and $16 \mathrm{~mm}$ with a standard deviation of $9.4 \mathrm{~mm}$. The aim of our simulator is to obtain a geometry resulting from the simulation that is as close as possible to the final position of the diaphragm. Fig. 5 shows a clear correlation between both meshes, with average displacements of $4.29 \mathrm{~mm}$ with a standard deviation of $3.6 \mathrm{~mm}$ for Subject 2, and $12 \mathrm{~mm}$ with a standard deviation of $6.7 \mathrm{~mm}$ for Subject 3 . The diaphragm contraction/relaxation modelling is good enough to simulate a realistic behaviour for liver access procedure training, but it would need to be further improved to be used in radiotherapy where precision is crucial.

The performance evaluations of Tables 1 and 2 show that the addition of the tensegrity constraint makes a slightly slower simulation, but it is still more than adequately fast. The recorded times were very similar. This demonstrates that, even in large models with hundreds of rigid links, the computational time is not massively increased.

Rib kinematics makes a considerable difference to the speed. This is a result of some large equations being solved to update the locations at each iteration. If a patient is observed to only be using the diaphragm and no rib action during respiration (as subject 1, 2 and 3), a large computational cost can be avoided. Another influencing factor on the speed of a simulation is the size of the mesh. Both virtual and patient models were tested with different resolutions. The evaluation shows an intuitive linear relationship between the number of vertices in the scene and the time taken in computation. 


\section{Conclusions}

We have presented here a novel methodology to simulate the behaviour of the diaphragm. It is based on a heterogeneous model composed of mass-spring and tensegrity elements. These latter elements are used to rigidify the tendinous tissue. The physiological boundary conditions have been carefully studied and applied to our model: the diaphragm motion follows more than one parameter, thus our model also takes into account the influence of the rib kinematics, the muscle natural contraction/relaxation and the motion of the sternum.

Our implementation runs in real-time and can be fine-tuned by adjusting the simulation parameters, e.g. if the patient is holding her breath or suddenly having a normal tidal breathing. The anatomy and the physiology can also be customized by generating patient-specific segmentations, mesh models and individual diaphragm actions. In the future, we aim to further validate our method and apply it to liver biopsy planning and rehearsal, as well as to monitoring lung motion in a radiotherapy context.

\section{References}

1. Shirato $\mathrm{H}$ et al (2006) Speed and amplitude of lung tumor motion precisely detected in fourdimensional setup and in real-time tumor-tracking radiotherapy. International Journal of Radiation Oncology, Biology, Physics 64(4):1229-1235

2. Rohlfing $\mathrm{T}$ et al (2004) Modeling liver motion and deformation during the respiratory cycle using intensity-based free-form registration of gated MR images. Medical Physics 31(3):427432

3. Siebenthal M, Szekely G, Lomax AJ, Cattin P (2007) Intersubject modelling of liver deformation during radiation therapy. In: MICCAI (1), pp. 659-666

4. Promayon E (1997) Modelling and simulation of the respiration. Ph.D. thesis, University Joseph Fourier of Grenoble

5. Zordan V et al (2004) Breathe easy : Model and control of simulated respiration for animation. In: ACM SIGGRAPH Symposium on Computer Animation

6. Villard PF, Bourne W, Bello F (2008) Modelling Organ Deformation Using Mass-Springs and Tensional Integrity. In: Proceedings of the 4th international Symposium on Biomedical Simulation, LNCS, vol. 5104, pp. 221-226

7. Ingber D (2000) Opposing views on tensegrity as a structural framework for understanding cell mechanics. J Appl Physiol 89:1663-1678

8. Whitelaw WA (1987) Shape and size of the human diaphragm in vivo. J Appl Physiol 62(1):180-186

9. Gauthier AP, Verbanck S, Estenne M, Segerbarth C, Macklem PT, Paiva M (1994) Threedimensional reconstruction of the in vivo human diaphragm shape at different lung volumes. J Appl Physiol 76(2):495-506

10. Aladin B, Rodarte J (1994) Inferences on passive diaphragm mechanics from gross anatomy. J Appl Physiol 77(5):2065-2070

11. Meier M (2005) Real-time deformable models for surgery simulation: a survey. Comput Methods Programs Biomed. 77(3):183-97

12. Nedel LP, Thalmann D (1998) Real time muscle deformations using mass-spring systems. In: CGI, pp. 156-165 
Interactive Simulation of Diaphragm Motion Through Muscle and Rib Kinematics

13. Didier AL, Villard PF, Bayle JY, Beuve M, Shariat B (2007) Breathing Thorax Simulation based on Pleura Behaviour and Rib Kinematics. In: IEEE Information Visualisation - MediVis, pp. 35-40 